\title{
Effect of Grammatical Gender of Language on Gender Stereotypes
}

\author{
Fengran Qiao ${ }^{1 *}$, Xinran Guo ${ }^{2 *}$, Jimin Yang ${ }^{3 *}$, Yinghua $\mathrm{Yu}^{4 *}$ \\ ${ }^{1}$ Faculty of Arts, University of British Columbia, Vancouver, V6T 1Z4, Canada. Email: qiaofengranzz@ gmail.com \\ ${ }^{2}$ Shenzhen College of International Education, Shenzhen, 518017, China. Email: awesomeaudsx@gmail.com \\ ${ }^{3}$ Maranatha High School, California, Pasadena 91105, America. Email: yangjordan70@gmail.com \\ ${ }^{4}$ Vanke Meisha Academy, Shenzhen, 518085, China.Email: ingrid.yu915@gmail.com
}

\begin{abstract}
The author noticed that in many languages such as Spanish and French are gendered in nature, specifically, nouns are divided into two types: masculine and feminine. From this, the author wondered if use of gendered language would tend to be associated with a higher level of gender stereotype. If the correlation between language and gender stereotypes is determined, then, the author wondered if the existence of grammatical gender in languages will influence gender stereotypes. Therefore, the author are proposing 2 studies exploring the relationship between gendered language and level of gender stereotype, as well as the relationship between the grammatical gender and gender stereotypes through four steps--elimination, conditioning, testing, data analysis.
\end{abstract}

Keywords: Gender stereotypes, Grammatical gender, language

\section{INTRODUCTION}

In the last few decades, gender stereotypes have been a popular topic which was hotly debated not only on social media but also in academic fields. According to Deaux and Lewis[1], gender stereotype refers to a set of shared beliefs about the traits, qualities, and tendencies associated with members of different sex categories. Gender stereotypes, as a subcategory of stereotypes formed by humans, are held by every individual since they are pervasively used by people to make quick interpretations about new information based on the knowledge and experiences they have in the past.

Gender stereotypes can be divided into two categories, explicit and implicit gender stereotypes. Explicit gender stereotypes are defined as the gender stereotypes that individuals are aware of and are able to express verbally. In contrast, implicit gender stereotypes are unconscious associations that people make between a social group (e.g., men) and a domain (e.g., science) or a trait (e.g., leadership). Additionally, there are four main components of gender stereotype, including the trait dimensions, role behaviors, occupations, and physical appearance. Most research focuses on the trait dimensions, traditionally known as masculine and feminine while now scholars prefer to use agency and communion respectively.
According to an abundance of studies that have been done, it is evident that a variety of verbal factors can make contributions to activating and reinforcing gender stereotypes, including reading the text written in masculine generics[2], using of Italian role nouns as prime words featured by semantic gender[3].

Besides verbal expression, grammar is another key component of linguistic elements while there is little gender stereotype research that draws attention to the grammatical aspect. In general, languages can be divided into two categories-gendered and genderless. Examples of gendered languages, languages with grammatical gender, are French, Spanish, German etc. Genderless languages include Chinese, English and Japanese.

Grammatical gender refers to the linguistic system which includes feminine and masculine categorization for nouns. Some languages like German also have a category of gender neutral, but we will only discuss languages that divide nouns into masculine and feminine in this experiment. For instance, "la rosa" (the rose, feminine, Spanish), "el sol" (the sun, masculine, Spanish). "La" and "El" are equivalent to "the" in English. The same works for "las" (the, plural, feminine) and "los" (the, plural, masculine). It seems intriguing how people in the past decided to assign a word into either masculine or feminine category. However, the masculine and feminine 
categories present in the Spanish grammatical system may create a self-explanatory system for people to make associations between noun and grammatical gender assigned to it through cultural practices and gender stereotyping. In this example, we can see that rose, a word with stereotypically feminine connotations of beauty and fragility is classified as a feminine noun while the sun, a word with stereotypically masculine connotations of boldness and defiance, is classified as a masculine noun. This can influence how people think about gender because if one's language links certain nouns with a gender, people could be influenced into linking the noun's connotations with that gender. However, there could be another possibility. Languages like Spanish and French assign gender to every noun, including those that don't have obvious gender connotations such as the table (la mesa, feminine), the bread (el pan, masculine), so this might actually weaken gender stereotypes. In this experiment, we will explore the relationship between grammatical gender and gender stereotypes.

\subsection{Specific Aims}

1. To test whether there is correlation between language and gender stereotypes.

2. To test whether the existence of grammatical gender in languages will influence gender stereotypes.

\section{PROPOSED STUDY 1}

Our hypothesis is that non-gendered language speakers (i.e., English) show less degree of gender stereotypes in the self-report scale compared to gendered language speakers (i.e., Spanish).

\section{Methods}

\section{Participants}

We invite 100 native Spanish speakers from Spain and 100 native English speakers from the US and ask them to complete the Gender Role Stereotype Scale (GRSS) which was developed by Mills et al.[4]. We record all scores then calculate the average score for each group.

\section{Prediction 1 and Possible Conclusion}

Our prediction 1 is that Spanish speakers score higher than English speakers on average.

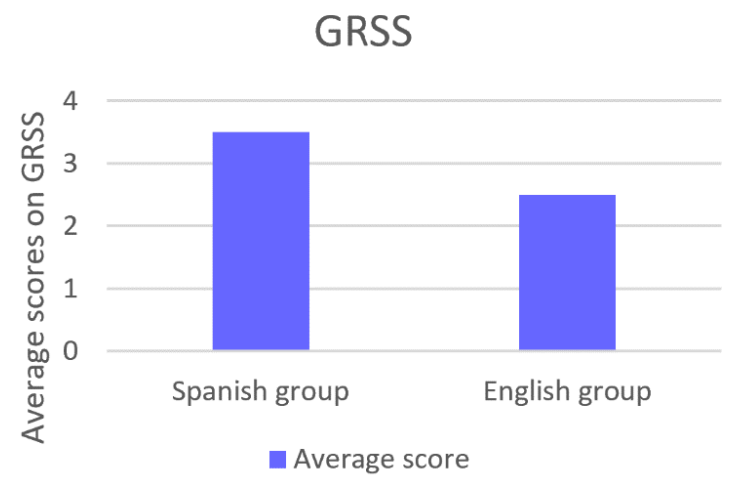

Figure 1 Prediction of the proposed study 1

If the results align with our prediction, it will suggest that gendered language tends to be associated with a higher level of gender stereotypes and we will conduct study 2 to find out if this relationship is a causal one. In contrast, if the result does not support the prediction, then the study 2 will not be conducted.

\section{PROPOSED STUDY 2}

The proposed study addresses the question of to what extent does grammatical gender influence people's gender stereotypes?

To address this question, we firstly attempt to tease apart the potential influence of cultural differences which will confound the relationship between language and gender stereotypes. Moreover, we will only focus on gender stereotypes on cisgender men and woman. People who are not on both ends of the gender spectrum are not included in this experiment. In the scope of this proposed study, strictly focusing on gender stereotypes on males and females would help our experiment to have a clearer structure.

\section{Method}

\section{Participants}

We will recruit 400 local Spanish English bilinguals who are selected from university campuses in California because Spanish is a gendered language while English is not. Since the experiment will be totally online, it is easy to include participants from a variety of universities. We choose California because it has the second highest concentration of Spanish speakers in the United States. As of 2010, 28.46\% $(9,696,638)$ of California residents aged 5 and older spoke Spanish at home as a primary language[5]. Also, California is the seventh most progressive state, and we attempt to select participants from a relatively educated and progressive environment because people who live in an extremely conservative environment might not be able to answer the questions in the following steps at all[6]. By choosing all participants from the same local area, we can tease apart the cultural factor from our study 2 since all the participants grew up 
in a relatively identical community and they share the same cultural background. Furthermore, we intentionally exclude students who study relevant majors like gender studies since they are very likely to be more conscious about gender stereotypes thus the questions we designed would not work well on them. Participants who take part in this experiment will be compensated with 5 dollars each.

\section{Step 1: baseline testing}

In this step, we aim to set the baseline value for the experiments by giving participants the Gender Role Stereotype Scale (GRSS), which detects gender stereotypes by using items that are easily understandable[4]. We attempt to use this questionnaire to measure people's existing gender stereotypes. This test is a self-report style scale, so it aims to test on participants' explicit gender stereotypes, which means that they are aware and conscious of their choices.

After collecting results from the questionnaire, we will eliminate the highest and the lowest scores and calculate the average of the remaining scores. Participants who have scores that are far off from the average score would be permitted into the next steps and the rest would be eliminated because we want to make sure that all our participants are generally all on the same starting line. The differences of their performance in the following steps could be attributed to the participants' existing gender stereotypes from experience rather than the effect of conditioning of experimental design.

Also, we will operate the step two weeks prior to step 2 and tell participants that this step is independent to the following experiment. All the questions in the scale tests are on genders, so it would be obvious to the participants that they are being tested on gender stereotypes. We do not want our participants to know that the following steps are also on gender stereotypes because that might make them more conscious when making choices and therefore hindering the experiment.

Furthermore, we put this step at first because a.) we can save the time and labor required to carry tests on the remaining participants and b.) the conditioning and testing might change participants' perception of genders and the results for this scale test will become inaccurate, so we can not put this test after the conditioning and testing.

\section{Step 2: conditioning}

We randomly assign the remaining participants into Group 1: Spanish group and Group 2: English group. Each group has the same number of people. Each group is given a short text in Spanish and English respectively. The length of the text would be about 10 minutes to read. The content in both texts would be completely the same and we would just alter the language. We would use backwards translation to ensure the content of text in two versions are the same, which can rule out the potential confound variable brought by the differences in information conveyed. We would invite a SpanishEnglish bilingual to translate the Spanish text into English and compare the translated version with the original English text to compare the differences. If the two versions differ in meanings, we will make alterations to the original Spanish text and invite another SpanishEnglish bilingual to do the translation to make the two versions as similar as possible. We will prepare a scientific text on the natural world because a.) a scientific, informative text would be written in an objective and formal tone so there is no confounding variables and; $b$.) we would include words with gendered connotations like the rose (i.e., la rosa, feminine word in Spanish); the sun (i.e., el sol, masculine word in Spanish); the moon (i.e., la luna, feminine word in Spanish). This might make the participants link a certain gender with their stereotypes (i.e., women are soft and tender; men are tough and confident)

\section{Step 3: testing phase}

Participants would be presented with a question to answer. Again, to eliminate demand characteristics and guessing behavior of participants when they give an answer to the question which would produce inaccurate data for the experiment, we attempt to prevent them from realizing the session is testing gender stereotypes. Thus, we will prepare a cover story and say that this question is used to test their logic skills. Also, we added in some details in the question to delicate the cover story and make our "logic skills" story become more plausible and deceptive. The 'logic' question we provide will be:

Question: a CEO of a stock company is driving a boy home from prom in a white SUV. When they arrived home, the boy rushed in to hug his dad. The dad offered the boy a drink and said that he was proud of everything he had achieved in high school. What might be the relationship between the boy and the CEO?

The standard answer would be mother. However, due to the Think Manager-Think Male effect, most participants would be likely to answer a male figure as the CEO[7] . This test is used to test our participants' gender stereotypes because women are stereotyped to be incapable of making big decisions and not suitable for leadership positions, so people don't usually think of women when they hear the word CEO. We will make this question a free-response question instead of a multiplechoice question because a.) this is an open-ended question and there is an infinite number of possible answers; b.) participants might make analysis and deductions based on the choices offered and therefore this way does not reflect their first thoughts.

\section{Step 4: data analysis}

We realize that participants might give all sorts of creative yet reasonable answers (e.g., this is a two-dad 
family; brother). Hence, we will process the data collected in Step 3 by dividing the responses into two categories- male characters and female characters (e.g., participants who answer "brother", "the other dad" and other male relatives will be put in the same categories). This is because participants who give male answers still assume that the CEO is a male figure in general. In addition, gender neutral responses such as "boss" or "another family member" will not be counted in the data processing.

Furthermore, we choose to not code every answer individually because there is no way to determine which answer shows more gender stereotype solely based on the answers for the question provided in Step 3 (i.e., we cannot distinguish the degree of gender stereotypes between a participant who answers "brother" and a participant who answers "uncle").

We will denote a few symbols for further calculation. I.) The total number of participants who register and complete the study as $T$; II.) The number of participants who were marked to be eliminated from participating in the following steps when they are doing Step 1 as $e$; III.) The number of people who answered the mother or female characters in Step 3 as $f$; IV.) The number of people who answered gender neutral characters in Step 3 as $n$; V.) The number of people who answer the father or male characters in Step 3 as $m$ (i.e., $T=e+f+n+m$ ). We will calculate $f /(T-e)$ for both groups and compare the results.

\section{Predictions 2 and Possible Conclusion}

Our prediction 2 is $f /(T-e)$ is bigger for the English group. This would mean that participants in the English group show less gender stereotypes since the gendered language used by the Spanish group will implicitly activate and reinforce the gender stereotypes. This might be because while completing the reading task, participants from the Spanish group are influenced by the connotations of the gendered nouns implicitly.

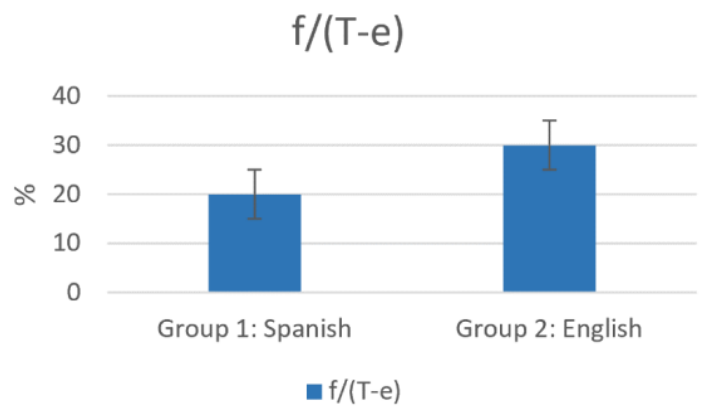

Figure 2 Prediction of proposed study 2

\section{Debriefing}

We told our participants in the first elimination step that the GRSS and the test they later do are not related while they in fact are, so we will need a debriefing part to inform them about it. We also told our participants in our testing step that the question was intended to test their logic skills while in fact it was about gender stereotypes, that would require us to debrief the participants too.

\section{IMPLICATIONS AND FURTHER STUDIES}

If the results of the proposed study support the hypothesis, this outcome would provide a brand-new perspective of how language influences our thoughts, especially on social thinking and interpersonal interactions, from a grammatical aspect. Alternatively, if the results are inconsistent with the prediction, there will be a few things to be further explored. Apart from the prediction 2 mentioned above, there are other two possible scenarios.

1. $f /(T-e)$ is bigger for the Spanish group. This would mean that participants in the Spanish group show less gender stereotypes. Take Spanish for an example, every noun is gendered, even the ones without gender connotations such as the table (la mesa, feminine), and the bread (el pan, masculine). By gendering those words with no clear gender connotations, it is possible that Spanish people might actually have weaker gender consciousness because they are so used to referring to random things in a certain gender that they don't strictly apply the stereotypes on people anymore.

2. $f /(T-e)$ for the two groups don't have significant differences. This shows that participants in the Spanish group and the English group show equal gender stereotypes. This might be because that language is influential enough to affect people's gender stereotypes.

Additionally, if the results are inconsistent with the prediction, there will be a few things to be further explored. For example, we may need to find more data for different languages to determine whether our hypothesis is incorrect or if our study is imprecise. Also, we might find participants from different places in a further study, not only from California, because participants from one location might give biased results. Finally, we might consider an additional study that will show participants images of objects and have them categorize these images as more alike to be male or female, to avoid possible confusions based on gendered word choice in a gendered language.

\section{CONCLUSION}

This experiment proposal is coming from pure curiosity on the interaction of language and thoughts, specifically testing for the relationship between language and gender stereotypes. Hence, the conclusion reached in this experiment should not be over interpreted into any political use. Furthermore, gender stereotypes could be understood as an information processing mechanism used 
by the human brain from a completely cognitive perspective, therefore has nothing to do with any political view.

\section{REFERENCES}

[1] Deaux, K., \& Lewis, L. L. (1984) Structure of gender stereotypes: Interrelationships among

components and gender label. Journal of Personality and Social Psychology, 46(5): 991-1004. https://doi.org/10.1037/0022-3514.46.5.991

[2] Kollmayer. M., Pfaffel, A., Schober, B., \& Brandt, L. (2018) Breaking away from the male

stereotype of a specialist: Gendered language affects performance in a thinking task. Frontiers in Psychology, 9: 985-985. https://doi.org/10.3389/fpsyg.2018.00985

[3] Cacciari, C., \& Padovani, R. (2007) Further evidence of gender stereotype priming in

language: Semantic facilitation and inhibition in italian role nouns. Applied Psycholinguistics, 28(2): 277-293.

https://doi.org/10.1017/S0142716407070142

[4] Mills, M. J., Culbertson, S. S., Huffman, A. H., \& Connell, A. R. (2012) Assessing gender

biases: Development and initial validation of the gender role stereotypes scale. Gender in Management, 27(8): 520-540. https://doi.org/10.1108/17542411211279715

[5] Internet Archive. (n.d.). California QuickFacts from the US Census Bureau. https://web.archive.org/web/20001109073000/http: //quickfacts.census.gov/qfd/states/06000.html

[6] World Population Review. (2021). Most Liberal States 2021. https://worldpopulationreview.com/staterankings/most-liberal-states

[7] Bosson, K. J., Vandello, J. A., \& Buckner, C. E. (2019) The psychology of sex and gender.

SAGE. ISBN: 9781506331324 